\title{
To uncover what is unknown or forgotten - cardiac magnetic resonance in the identification of vascular pathologies in patients after tetralogy of Fallot repair
}

\author{
Mateusz Śpiewak ${ }^{1,2}$, Łukasz A. Małek ${ }^{1,3}$, Elżbieta K. Biernacka ${ }^{4}$, Mirosław Kowalski ${ }^{4}$, Piotr Hoffman ${ }^{4}$, \\ Marcin Demkow ${ }^{2}$, Jolanta Miśko', Witold Rużyłło ${ }^{5}$, Magdalena Marczak ${ }^{1}$ \\ ${ }^{1}$ Magnetic Resonance Unit, Department of Radiology, Institute of Cardiology, Warsaw, Poland \\ ${ }^{2}$ Department of Coronary Artery Disease and Structural Heart Diseases, Institute of Cardiology, Warsaw, Poland \\ ${ }^{3}$ Department of Interventional Cardiology and Angiology, Institute of Cardiology, Warsaw, Poland \\ ${ }^{4}$ Department of Congenital Heart Diseases, Institute of Cardiology, Warsaw, Poland \\ ${ }^{5}$ Institute of Cardiology, Warsaw, Poland
}

Postep Kardiol Inter 2014; 10, 2 (36): 104-109

DOI: $10.5114 /$ pwki.2014.43516

\section{Abstract}

Introduction: In patients with repaired tetralogy of Fallot (TOF), various pathologies of the vascular system (both arterial and venous) may be present as a result of the previous therapeutic procedures or due to the congenital disease itself. Because of the limited diagnostic capabilities in the past, lacking surgical reports of patients operated on several decades ago and/or a long time since a corrective procedure, some of these pathologies/anomalies may remain unknown.

Aim: To identify selected vascular pathologies with the use of cardiac magnetic resonance in patients after TOF repair.

Material and methods: We included 208 consecutive patients (median age 24.9 years, interquartile range 20.5-36.7; 126 (60.6\%) males) with repaired TOF undergoing cardiac magnetic resonance (CMR) imaging.

Results: Significant unexpected vascular pathologies/anomalies were found in 30 patients (14.4\%) and included: uni- or bilateral occlusion of the subclavian artery $(n=20)$, persistent left superior vena cava $(n=7$, in 1 case draining into the left atrium), occluded subclavian vein $(n=1)$, and interruption of the inferior vena cava $(n=2)$. Additionally, 1 patient with the left subclavian artery occluded had an occlusion of the brachiocephalic vein. In none of the patients was the information about the uncovered pathology/ anomaly present either in the referral information or in the present medical history.

Conclusions: The CMR in patients with repaired TOF may uncover some pathologies/anomalies which were unknown or forgotten at the time of patients' referral for the study, and which may have a significant impact on patient management.

Key words: cardiac magnetic resonance, tetralogy of Fallot, Blalock-Taussig shunt, subclavian artery occlusion, persistent left superior vena cava, interruption of the inferior vena cava.

\section{Introduction}

Tetralogy of Fallot (TOF) is the most common cyanotic congenital heart disease [1]. Owing to the progress in the surgical treatment, the majority of patients reach adulthood and constitute a continuously growing population $[1,2]$. However, many patients long after the primary repair suffer from late consequences, e.g. pulmonary regurgitation, pulmonary stenosis, residual ventricular septal defect, tricuspid regurgitation, and others. Therefore, the majority of patients require re-interventions (either surgical or percutaneous) to have these lesions treated [1,
3-5]. Moreover, due to atrial or ventricular arrhythmias, or conduction disturbances, a substantial group of patients with repaired TOF develop indications for pacemataneker or cardioverter-defibrillator implantation, or percutaneous radiofrequency catheter ablation. Additionally, since most such patients reach adulthood, they require interventions not only due to the underlying congenital pathology but also due to acquired cardiac diseases such as obstructive coronary artery disease.

Any re-intervention in a previously operated patient is a great challenge for a surgeon and/or an interventional

\section{Corresponding author:}

Mateusz Śpiewak MD, PhD, Magnetic Resonance Unit, Department of Radiology, Institute of Cardiology, 42 Alpejska St, 04-682 Warsaw, Poland, phone: +48 2234346 57, fax: +48 2234345 52, e-mail: mspiewak@ikard.pl

Received: 24.03.2014, accepted: 11.04.2014. 
cardiologist. One of the most crucial elements of the successful percutaneous treatment is the appropriate vascular access which provides suitable conditions for device maneuvers as well as guaranteeing patients' safety. Therefore, pre-procedural patients' evaluation is of particular importance.

According to the current European guidelines on management of patients with congenital heart diseases, all patients with repaired TOF should undergo cardiac magnetic resonance $(C M R)$ imaging [1]. The CMR enables not only accurate and reproducible measurements of ventricular size and function to be done, but also is considered the reference standard for the quantitative assessment of pulmonary regurgitation $[1,6,7]$. Additionally, CMR is an attractive emerging technique for the visualization of great vessels $[1,6]$. Therefore, CMR is being increasingly used in this patient population, becoming a part of the standard patient management [8].

In patients with repaired TOF, various pathologies of the vascular system (both arterial and venous) may be present as a result of the previous therapeutic procedures (e.g. occlusion of the subclavian artery as a consequence of the previous Blalock-Taussig shunt) or due to the congenital disease itself. Because of the limited diagnostic capabilities in the past, lacking surgical reports of patients operated on several decades ago and/or a long time since a corrective procedure, some of these pathologies/anomalies may remain unknown to a treating physician. Nevertheless, they may affect patients' treatment.

\section{Aim}

Our hypothesis was that CMR may uncover unknown pathologies and anomalies in patients after TOF repair, which may impact the invasive management of this population.

\section{Material and methods}

\section{Patients}

Patients after TOF repair were considered eligible for the study if they had at least one CMR study including contrast-enhanced magnetic resonance angiography performed in the analyzed period (from June 2008 to end of December 2012). Patients with artifacts in CMR images precluding reliable assessment were excluded. Each patient and/or parent/guardian gave written informed consent for CMR study. The approval for the use of the data in the study was granted by the institutional Ethics Committee.

\section{Cardiac magnetic resonance}

All CMR scans were performed as part of a normal assessment as described previously [8]. In brief, all patients underwent standard CMR imaging on a $1.5 \mathrm{~T}$ scanner (Avanto, Siemens, Erlangen, Germany). The protocol encompassed, among others, the following acquisitions: transaxial, coronal, and sagittal turbo spin echo images (half-Fournier single-shot turbo spin-echo - HASTE) and balanced steady-state free precession (both single-shot and cine) images, as well as contrast-enhanced magnetic resonance angiography. The presence of unexpected findings was evaluated on each of the available images.

As in a normal clinical scenario, findings of each CMR scan were regularly assessed by a team with experience in congenital heart diseases (CHD): a physician with experience in CMR imaging, a clinician, an interventional cardiologist, and an experienced radiologist. The decision on the relevance of CMR findings was reached by consensus.

The findings of CMR scans were compared to the patients' present medical history, the referral information, and other reports available at the time of CMR imaging. The CMR findings were categorized as: (1) expected, when the finding was known from the available records, (2) unexpected, when CMR revealed a new finding which was not included in a patient's medical history or referral information. We analyzed only unexpected findings potentially influencing patient management. Incidental findings with no significant impact on patient management were not considered.

We did not aim to compare either ventricular size and function or the severity of pulmonary regurgitation between CMR and echocardiography; thus those parameters were not analyzed in the present study. In other words, any discrepancies in the ventricular size or the degree of pulmonary regurgitation between CMR and echocardiography were not considered as unexpected findings.

\section{Statistical analysis}

After checking for normal distribution with the Kolmogorov-Smirnov test, continuous variables were compared using either Student's $t$-test or the Mann-Whitney test as appropriate. Categorical variables were compared using the $\chi^{2}$ test or the Fisher exact test. A two-sided $p$-value $<0.05$ was considered significant. All statistical analyses were performed using MedCalc 12.1.4.0 software (MedCalc; Mariakerke, Belgium).

\section{Results}

Two hundred and thirteen patients underwent $C M R$ in the period analyzed. However, 5 of them were excluded due to incomplete CMR (due to anxiety caused by claustrophobia leading to procedure termination before the whole CMR protocol, including angiography, was completed). Two hundred and eight patients were included in the final analysis (median age 24.9 years, interquartile range $20.5-36.7 ; 126$ (60.6\%) males).

Overall, significant venous or arterial vascular pathologies/anomalies were found in 30 patients (14.4\%) 
Table I. Details of unexpected findings uncovered in CMR imaging

\begin{tabular}{|c|c|}
\hline $\begin{array}{l}\text { Vascular pathologies/anomalies found with } \\
\text { the use of CMR }\end{array}$ & Details of the vascular pathology/anomaly \\
\hline \multirow[t]{3}{*}{ Subclavian artery occlusion $(n=20)$} & $\begin{array}{l}\text { Left-sided occlusion }(n=12) \text { : } \\
\text { - Left-sided Blalock-Taussig shunt in the history }(n=11) \\
\text { - Medical reports were lost and were unobtainable and/or no information on previous } \\
\text { Blalock-Taussig shunt was given in the available medical data }(n=1)\end{array}$ \\
\hline & $\begin{array}{l}\text { Right-sided occlusion }(n=6) \text { : } \\
\text { - Right-sided Blalock-Taussig shunt in the history }(n=4) \\
\text { - Medical reports were lost and were unobtainable and/or no information on previous } \\
\text { Blalock-Taussig shunt was given in the available medical data }(n=2)\end{array}$ \\
\hline & $\begin{array}{l}\text { Bilateral occlusion }(n=2) \text { : } \\
\text { - Bilateral Blalock-Taussig shunt in the history }(n=2)\end{array}$ \\
\hline Persistent left superior vena cava $(n=7)$ & $\begin{array}{l}\text { - Draining into the coronary sinus }(n=6) \\
\text { - Draining into the left atrium }(n=1)\end{array}$ \\
\hline Interrupted inferior vena cava $(n=2)$ & $\begin{array}{l}\text { With azygos/hemiazygos continuation }(n=1) \text { : } \\
\text { - With collateral circulation through superficial collateral veins in the abdominal wall } \\
(n=1) \text {; additionally, this patient had right-sided subclavian artery occlusion }\end{array}$ \\
\hline Right-sided subclavian vein occlusion $(n=1)$ & \\
\hline
\end{tabular}

Table II. Comparison of clinical characteristics of patients with unexpected findings in CMR imaging versus those without any significant vascular pathologies/anomalies

\begin{tabular}{|c|c|c|c|}
\hline Parameter & $\begin{array}{l}\text { Unexpected findings (+) } \\
\qquad n=30\end{array}$ & $\begin{array}{l}\text { Unexpected findings }(-) \\
\qquad n=178\end{array}$ & Value of $p$ \\
\hline Age [years] & $41.9 \pm 12.2$ & $27.1 \pm 9.5$ & $<0.0001$ \\
\hline Males, $n(\%)$ & $21 / 30(70.0 \%)$ & $105 / 178(59.0 \%)$ & 0.35 \\
\hline Age at TOF repair [years] & $12.0(6.6-33.0)$ & $3.8(2.3-6.2)$ & $<0.0001$ \\
\hline Time since TOF repair [years] & $20.8(16.5-28.7)$ & $19.3(15.7-23.6)$ & 0.32 \\
\hline $\begin{array}{l}\text { Number of previous cardiothoracic } \\
\text { operations }\end{array}$ & $2(1-3)$ & $1(1-2)$ & 0.0001 \\
\hline Previous palliative shunt*: & $19 / 30(63.3 \%)$ & $39 / 177(22.0 \%)$ & $<0.0001$ \\
\hline Blalock-Taussig shunt & $19 / 30(63.3 \%)$ & $37 / 177(20.9 \%)$ & $<0.0001$ \\
\hline Others & $0(0 \%)$ & $2 / 177(1.1 \%)$ & 0.5 \\
\hline
\end{tabular}

Data are presented as numbers and percentages, means and standard deviations, or medians with interquartile ranges. ${ }^{*}$ Data available for 207 patients

and included (Table I): (A) occluded subclavian artery ( $n$ $=20$; in 12 patients the occlusion of the left subclavian artery was found, in 6 patients the right subclavian artery was occluded, and 2 patients had bilateral occlusion), (B) persistent left superior vena cava ( $n=7$; draining into the coronary sinus in 6 patients, in 1 patient it drained into the left atrium with non-significant right-toleft shunting), (C) interruption of the inferior vena cava ( $n=2$; 1 patient had additionally right-sided subclavian artery occlusion) and (D) occlu ed subclavian vein $(n=1)$ (Figure 1). Additionally, 1 patient with occlusion of the left subclavian artery had occlusion of the brachiocephalic vein. In none of the patients was the information about the uncovered pathology/anomaly present either in the referral information or in the present medical history. All those findings were categorized as unexpected. The characteristics of patients with the above-mentioned pa- thologies/anomalies are presented in Table I. All patients with occlusion of the subclavian artery were asymptomatic, i.e. none of them complained of decreased muscle strength or impaired function of the upper extremities.

The comparison of clinical characteristics of patients with unexpected findings in CMR imaging versus those without any significant vascular pathologies/anomalies is outlined in Table II, and the comparison of the imaging findings in Table III. Those with unexpected findings were significantly older at the time of CMR imaging as well as at the primary repair (Table II). As expected, both the use of palliative shunts in history, and the number of previous cardiothoracic operations were higher in patients with unexpected findings.

The comparison of the imaging findings revealed lower mean pulmonary regurgitation fraction, lower proportion of patients with significant pulmonary regur- 

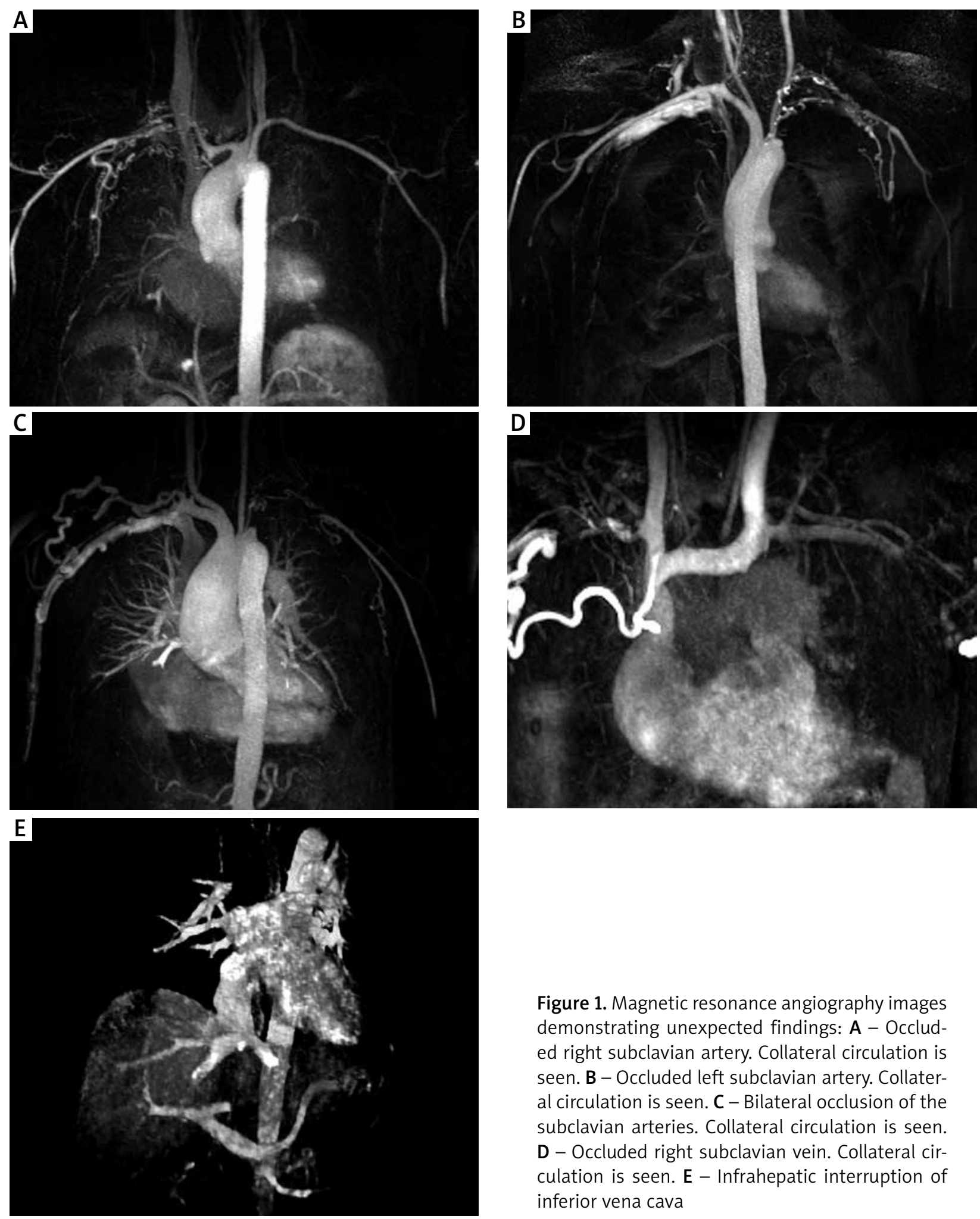

Figure 1. Magnetic resonance angiography images demonstrating unexpected findings: A - Occluded right subclavian artery. Collateral circulation is seen. B - Occluded left subclavian artery. Collateral circulation is seen. $\mathbf{C}$ - Bilateral occlusion of the subclavian arteries. Collateral circulation is seen. D - Occluded right subclavian vein. Collateral circulation is seen. $\mathbf{E}$ - Infrahepatic interruption of inferior vena cava

gitation, and a trend toward a smaller right ventricular volume in the group with unexpected findings (Table III). Patients without unexpected findings had higher rightand left-ventricular ejection fractions, and a smaller left ventricular volume (Table III).

\section{Discussion}

We demonstrated that CMR uncovered significant pathologies/anomalies of the vascular system which remained unknown at the time of the patient being referred for the study. Each of the pathologies/anomalies defined 
as significant may potentially affect patient management. The majority of unexpected findings were occluded subclavian arteries (overall in 20 patients) associated with the use of the Blalock-Taussig shunt in history. This fact explains the higher age at TOF repair in this group of patients - the majority of these patients remained palliated prior to corrective surgery (Table I). The occlusion of the subclavian artery, although compensated by collateral flow and therefore causing no symptoms with regard to the function of the upper extremity, may lead to subclavian artery steal syndrome causing recurrent syncope, which triggers extensive diagnostic work-up of syncope in order to exclude cardiogenic causes $[1,9]$. Since unexplained syncope may herald an increased risk for ventricular arrhythmias and poorer outcome in patients with repaired TOF, the identification of each possible cause of syncope in this population is of paramount importance [1]. In patients with coronary artery disease, the occluded subclavian artery precludes transradial access for coronary angiography and/or percutaneous coronary interventions, and also may limit the use of the internal mammary artery (originating from the subclavian artery) as a conduit during coronary artery grafting procedures. Moreover, coronary subclavian steal syndrome may occur in the case when the internal mammary artery was used as a graft, and occlusion or stenosis of the subclavian artery develops [10].

Persistent left superior vena cava is the most common variant of systemic venous return. Its prevalence ranges from about $0.5 \%$ of the general population, up to $10 \%$ of patients with congenital heart defects [11]. In the majority of patients, it drains into the right atrium, via the coronary sinus. Therefore, the presence of left superior vena cava is usually a benign anomaly causing no symptoms and be- ing unrecognized throughout the patient's life. However, when it drains into the left atrium it may cause significant right-to-left shunting [11]. In patients undergoing cardiac surgery, persistent left superior vena cava may cause unexpected complications during perioperative and postoperative periods [12]. Additionally, persistent left superior vena cava draining into the coronary sinus may turn out to be problematic in several other clinical scenarios such as pacemaker implantation, cannulation of central veins, and catheter ablation [11, 13-15]. The same potential problems apply to occluded brachiocephalic vein or subclavian vein as well as congenital interruption of the inferior vena cava seen in our patients. Patients with repaired TOF are subjected to repeated invasive procedures including pacemaker/cardioverter-defibrillator implantation, percutaneous pulmonary valve implantation, catheter ablation for ventricular and atrial arrhythmias, as well as reoperations for pulmonary valve incompetence or stenosis, residual ventricular septal defect or tricuspid regurgitation. Thus, the awareness of the presence of these pathologies/anomalies is of particular importance for patients' safety and procedural success.

Interestingly, patients with unexpected findings had smaller pulmonary regurgitation when compared to those without unexpected findings in CMR imaging (Table III). It can be speculated that this may be attributable to older age at TOF repair in those with unexpected findings. On the other hand, both right- and left-ventricular ejection fraction was higher in patients without unexpected findings (Table III).

According to the current guidelines, CMR is found superior to echocardiography in various indications in patients with congenital heart diseases $[1,6]$. Due to the

Table III. Comparison of imaging findings

\begin{tabular}{|c|c|c|c|}
\hline Variables & $\begin{array}{l}\text { Unexpected findings ( }+ \text { ) } \\
\qquad n=30\end{array}$ & $\begin{array}{l}\text { Unexpected findings }(-) \\
n=178\end{array}$ & Value of $p$ \\
\hline $\operatorname{RVEDV~}\left[\mathrm{ml} / \mathrm{m}^{2}\right]$ & $142.7 \pm 42.1$ & $157.1 \pm 44.2$ & 0.099 \\
\hline $\operatorname{RVESV}\left[\mathrm{ml} / \mathrm{m}^{2}\right]$ & $81.8 \pm 32.4$ & $84.4 \pm 32.0$ & 0.69 \\
\hline $\operatorname{RVSV}\left[\mathrm{ml} / \mathrm{m}^{2}\right]$ & $62.4 \pm 18.4$ & $72.2 \pm 19.7$ & 0.01 \\
\hline RVEF [\%] & $43.1 \pm 7.9$ & $47.3 \pm 8.3$ & 0.01 \\
\hline RVM $\left[\mathrm{g} / \mathrm{m}^{2}\right]$ & $30.2 \pm 9.6$ & $30.3 \pm 9.1$ & 0.95 \\
\hline LVEDV $\left[\mathrm{ml} / \mathrm{m}^{2}\right]$ & $96.4 \pm 25.0$ & $86.8 \pm 18.1$ & 0.05 \\
\hline LVESV $\left[\mathrm{ml} / \mathrm{m}^{2}\right]$ & $45.1 \pm 17.1$ & $37.4 \pm 11.4$ & 0.02 \\
\hline LVEF [\%] & $53.9 \pm 7.7$ & $57.5 \pm 6.7$ & 0.008 \\
\hline $\operatorname{LVM}\left[\mathrm{g} / \mathrm{m}^{2}\right]$ & $61.0 \pm 14.0$ & $55.3 \pm 14.3$ & 0.04 \\
\hline $\operatorname{PRF}[\%]^{*}$ & $13(0-28)$ & $29(16-40)$ & 0.001 \\
\hline Significant PR (PRF $\geq 20 \%)^{*}$ & $12 / 28(42.9 \%)$ & $121 / 174(69.5 \%)$ & 0.01 \\
\hline Peak RVOT gradient [mm Hg] & $20(4-37)$ & $20(9-34)$ & 0.75 \\
\hline Significant RVOT gradient $(\geq 30 \mathrm{~mm} \mathrm{Hg})^{\#}$ & $12 / 29(41.4 \%)$ & $53 / 177(29.9 \%)$ & 0.31 \\
\hline
\end{tabular}

Data are presented as numbers and percentages, means and standard deviations, or medians with interquartile ranges. *Data available for 202 patients. \#Data available for 206 patients 
limitations of echocardiography, some of the pathologies or anomalies which are not seen on echocardiography are easily found in CMR. Although CMR is found superior to echocardiography in various clinical scenarios, echocardiography remains the imaging modality of choice not only in patients with TOF but also in all patients with CHD [1]. The CMR is, however, complementary to echocardiography and provides a wealth of data influencing patient management. Considering its significant impact on patient management, CMR should be regarded as an indispensable facility in every center for CHD patients [6].

It is likely that at least some if not all of the pathologies or anomalies found in CMR were known at the time of the primary repair or subsequent reoperations but either they were not included in the medical history, or medical reports were lost and the data were unobtainable. Unknown details of medical history of patients operated on over 20 or 30 years ago is a common issue in populations of patients with repaired TOF $[8,16]$. The CMR may be a partial remedy for this problem providing medical information which was lost during the patient's life. We did not perform whole body angiography; thus vascular pathologies/anomalies in other localizations such as femoral vein occlusion associated with repeated catheterizations cannot be excluded.

\section{Conclusions}

We demonstrated that CMR imaging in patients with repaired TOF may uncover some pathologies/anomalies which were unknown or forgotten at the time of patients' referral for the study, and which may have a significant impact on patient management. These findings confirmed the usefulness of routine CMR studies in this population.

\section{References}

1. Baumgartner H, Bonhoeffer P, De Groot NM, et al. ESC Guidelines for the management of grown-up congenital heart disease (new version 2010). Eur Heart J 2010; 31: 2915-57.

2. Go AS, Mozaffarian D, Roger VL, et al. Heart disease and stroke statistics - 2013 update: a report from the American Heart Association. Circulation 2013; 127: e6-245.

3. Ruzyllo W, Wlodarska EK, Demkow M, et al. Transvascular pulmonary valve implantation - early results of the first Polish experience. Postep Kardiol Inter 2009; 5: 1-6.

4. Ruzyllo W, Demkow M, Wlodarska EK, et al. POL-PAVTI - Polish report on transcatheter pulmonary artery valve implantation of Melody-Medtronic prosthesis in the first 14 patients in Poland. Postep Kardiol Inter 2009; 5: 137-43.

5. Fiszer R, Szkutnik M, Kusa J, Białkowski J. Experience in percutaneous atrial septal defects closure after previous cardiac surgery. Postep Kardiol Inter 2008; 4: 129-32.

6. Kilner PJ, Geva T, Kaemmerer H, et al. Recommendations for cardiovascular magnetic resonance in adults with congenital heart disease from the respective working groups of the European Society of Cardiology. Eur Heart J 2010; 31: 794-805.
7. Geva T. Repaired tetralogy of Fallot: the roles of cardiovascular magnetic resonance in evaluating pathophysiology and for pulmonary valve replacement decision support. J Cardiovasc Magn Reson 2011; 13: 9.

8. Spiewak M, Malek LA, Petryka J, et al. Repaired tetralogy of Fallot: ratio of right ventricular volume to left ventricular volume as a marker of right ventricular dilatation. Radiology 2012; 265 : 78-86.

9. Kurlan R, Krall RL, Deweese JA. Vertebrobasilar ischemia after total repair of tetralogy of Fallot: significance of subclavian steal created by Blalock-Taussig anastomosis. Vertebrobasilar ischemia after correction of tetralogy of Fallot. Stroke 1984; 15: 359-62.

10. Chai P, Dubowitz M, Mohiaddin R. Coronary-subclavian steal syndrome demonstrated by cardiovascular magnetic resonance. Circulation 2006; 113: e930-1.

11. Irwin RB, Greaves M, Schmitt M. Left superior vena cava: revisited. Eur Heart J Cardiovasc Imaging 2012; 13: 284-91.

12. Giuliani-Poncini C, Perez M, Cotting J, et al. Persistent left superior vena cava in cardiac congenital surgery. Pediatr Cardiol 2014; 35: 71-6.

13. Walsh EP, Cecchin F. Arrhythmias in adult patients with congenital heart disease. Circulation 2007; 115: 534-45.

14. Polewczyk A, Kutarski A, Czekajska-Chehab E, et al. Complications of permanent cardiac pacing in patients with persistent left superior vena cava. Cardiol J 2014; doi: 10.5603/CJ.a2014.0006.

15. Majewski J, Karkowski G, Miszalski-Jamka T, et al. Ablation of slow pathway in a patient with persistent left superior vena cava. Kardiol Pol 2013; 71: 509-11.

16. Wald RM, Redington AN, Pereira A, et al. Refining the assessment of pulmonary regurgitation in adults after tetralogy of Fallot repair: should we be measuring regurgitant fraction or regurgitant volume? Eur Heart J 2009; 30: 356-61. 